\title{
The Relationship Between Dependent Personality Disorder and Emotional Difficulties with Distress in Young Sa'adat Abad Couples in Tehran
}

\author{
Seyedeh Tayebe Zamani \\ Department of psychology ,college of human sciences ,Islamic Azad university of Boqan Branch ,West \\ Azarbayjan ,Iran
}

\begin{abstract}
The purpose of this study was to determine the relationship between dependent personality disorder and emotional adjustment difficulties with endurance distress in young couples in Sa'adat Abad. Regarding the title of the paper, the chosen methodology was descriptive. The statistical population of the study was all young couples of Sa'adat Abad in 2017. The statistical sample of this study was Morgan table, 80 couples ( 40 women and 40 men) residing in Sa'adat Abad district of Tehran, ranging from 25 to 35 years old, with marital life less than 5 years old, were sampled by random sampling methodology. Research instruments were Distress Tolerance Questionnaire (DTS), Cognitive Emotion Regulation Questionnaire (CERQ-p) and Dependent Personality Index (DPR-R). The results of the study indicated that there was a significant relationship between dependent personality disorder and distress tolerance in the young couples of Sa'adat Abad in Tehran. There was a significant negative correlation between emotional adjustment and distress tolerance in young couples in Sa'adat Abad. Also, there was a significant positive relationship between relying on others, the need for relationships and tolerance of distress in young couples in Sa'adat Abad. There was a significant negative relationship between the lack of acceptance of emotional responses, the difficulty in performing purposeful behavior, difficulty in controlling impulse, lack of emotional awareness, limited access to emotional regulation strategies, lack of clarity and distress in young couples in Sa'adat Abad.
\end{abstract}

Keywords: Dependent Personality Disorder, Emotional Adjustment Difficulties, Distress Tolerance.

DOI: $10.7176 / \mathrm{RHSS} / 9-12-07$

Publication date:June $30^{\text {th }} 2019$

\section{Statement of the problem}

Personality disorder involves a heterogeneity of disorder, and when it is recognized that people with abnormal and invasive traits persist throughout their life, these attributes may cause distress in the individual. Personality disorder is a pattern of sustainability of internal behavior and experience that is significantly contradictory to cultural expectations, is inclusive and uncompromising, begins in adolescence or adulthood, is sustained over time and is distressing. Or disruption (Seyyed Mahdavi Agdam et al., 2013: 210). The regulation of excitement is considered as the process by which individuals exchange their excitement for responding to the intentional and unhealthy environmental expectations (Aldao et al., 2010). Defeat in the setting of excitement is called normal. Thoughts of emotion are defined as maladaptive responses to emotions, which include the lack of acceptance responses, the difficulty in controlling behaviors in emotional distress, and the defect in the functional use of emotions as information (Graz \& Romer, 2004). Distress tolerance is one of the common structures for research on emotional disorder. In fact, distress tolerance is a variable of individual differences that refers to the capacity of experience and resistance to emotional distress. Tolerance of distress is increasingly seen as an important structure in the development of new insights on the initiation and maintenance of mental health as well as prevention and treatment. People with low endurance distress are involved in behavioral disorder in an erroneous attempt to deal with their negative emotions and seek to relieve their emotional pain by addressing some destructive behaviors such as consuming drugs. A thrilling coping strategy such as drinking alcohol and other substances may result in people getting rid of negative emotions quickly. This strategy, especially for those with low endurance distress, is considered appropriate (Azizi et al., 2010). Many factors can affect the quality of relationships between husband and wife. One of the most important of them is personality, and most importantly, one must be careful about personality disorders. Personality is one of the determinants of the reactions of situations that are stressful (Mohammadi \& Alipour, 2014). Personality disorder is common and problematic. 10 to 20 percent of the population are suffering from a type of personality disorder (Khodabakhsh, 1392). Therefore, according to the presented questions, the researcher is seeking to answer the question whether there is a relationship between dependent personality disorder in young couples in Sa'adat Abad.

\section{Research objectives}

The main objectives of the research

Determining the Relationship between Affective Personality Disorder and Distress Tolerance in Young Couples 
in Sa'adat Abad, Tehran.

Determining the Relationship Between Difficulties of Tuning and Extreme Tolerance in Young Couples in Sa'adat Abad, Tehran.

\section{Sub-goals}

Determining the relationship between relying on others and enduring distress in young couples in Sa'adat Abad, Tehran.

Determining the Relationship between Need for Relationships and Distress in Young Sa'adat Abad Couples in Tehran.

Determining the Relationship between Dismissal of Emotional Responses and Distress Tolerance in Young Couples in Sa'adat Abad, Tehran.

Determining the Relationship Between Difficulty in Targeted Behavior and Distress Tolerance in Young Couples in Sa'adat Abad, Tehran.

Determining the Relationship Between Difficulty Controlling Impulse and Distress Tolerance in Young Couples in Sa'adat Abad Tehran.

Determining the Relationship between Lack of Emotional Knowledge and Distress Tolerance in Young Couples in Sa'adat Abad, Tehran.

Determining the Relationship Between Difficulties of Tuning and Extreme Tolerance in Young Couples in Sa'adat Abad, Tehran.

Determining the relationship between limited access to emotional adjustment strategies and distress tolerance in young couples in Saadat Abad, Tehran.

Determining the Relationship between Emotional Deprivation and Distress Tolerance in Young Couples in Sa'adat Abad, Tehran.

\section{Research hypotheses}

Main research hypotheses:

There is a significant relationship between afflicted personality disorder and distress tolerance in the young couples of Sa'adat Abad in Tehran.

There is a significant relationship between the difficulties of emotion regulation and distress tolerance in the young couples of Saadat Abad in Tehran.

\section{Sub-hypotheses:}

There is a significant relationship between relying on others and enduring distress in young couples in Sa'adat Abad.

There is a significant relationship between the need for relationships and distress in young couples in Sa'adat Abad. There is a significant relationship between the lack of acceptance of emotional responses and endurance distress in young couples in Sa'adat Abad.

There is a significant relationship between the difficulty in targeting behavior and tolerance of distress in young couples in Sa'adat Abad.

There is a significant relationship between difficulty in controlling impulse and endurance distress in young couples of Sa'adat Abad.

There is a significant relationship between lack of emotional awareness and tolerance of distress in young couples in Sa'adat Abad.

There is a significant relationship between limited access to emotional adjustment strategies and distress tolerance in young couples in Sa'adat Abad.

There is a significant relationship between lack of clarity of emotion and tolerance of distress in young couples in Sa'adat Abad.

Operational definition:

Distress tolerance

In this research, stress tolerance is a scorecard that the person undergoing testing receives a 15 -item questionnaire of distress tolerance (DTS).

\section{Emotion context:}

In this research, emotional regulation is the score that a person undergoing the test of emotion regulation questionnaire (CERQ), set by Garnovskii, Kraiyev (2001).

\section{Dependent Personality Disorder:}

In this research, affiliated personality disorder is defined as the score that a person undergoing testing from an 
Associated Personality Disorder Questionnaire (DPI-R).

\section{Emotional regulation ideas \\ James Lange's Theory}

Based on this theory, exciting situations trigger specific physiological responses such as trembling, sweating and increased heart rate. It also calls for behaviors such as knitting or fighting. The brain from the muscles and organs that make up these reactions has a sensory response and this is a feedback that gives you emotional feelings. James says our emotional feelings are based on the practicality that comes from us and we receive a sense of muscle and inner organs. So, when we find ourselves trembling and nauseous, we experience fear. According to this theory, the excitements that are felt are perceptions of physical transformations. According to this theory, what we are experiencing excitely is a tag that we give our answers to. For example, since we are fleeing, then we are afraid. (Atkinson et al., 1992, translated by Barahini et al., 2003)

\section{Activation theory}

This theory is mainly proposed by Schleswig-Holstein. Instead of treating excitement as a particular and qualitatively different state than the other states, this theory determines the location of emotional behavior on a continuum that embraces all behaviors. The lower part of the continuum, which is called the general level of activity, is in a state of sleep, the middle sections are devoted to intense attention and the upper part to intense emotions.

\section{The basic concepts of Alice's theory \\ A) The theory of personality}

Ellis looks at the character from three physiological, social and psychological points of view.

Physiological basis: Alice believes that humans inherently have exceptional and powerful biological tendencies for thinking and action in a particular way, which may be rational or illogical. Alice understands the individual's ability to think in terms of family and cultural environment where one grows

Social basis: Alice accepts that human being is a social being, and believes that man must behave in a community in accordance with his or her own expectations and others, and that he is not self-sufficient or self-sufficient. Alice believes that by insisting on the attitude of others towards herself and expressing this attitude as urgently needed, she is a dysfunctional state of the soul.

Psychological basis: Alice does not accept the instinct in the classical sense, and is more inclined to agree with Maslow on human desires. He admits that man is reluctant to love and love, care and attention to dreams, and avoids hatred and frustration. Therefore, when an activating event occurs for an individual, he may have two opposing perceptions based on his intrinsic desires. One logical and rational thoughts, and second, irrational and irrational thoughts (Dohl Bay, 1981, Tabrizi, 2006).

\section{Self-regulatory approach to distress}

The first time that Canon discovered the excitement, referring to the fact that such an answer is rooted in the angry and angry feelings, it is called the response from an urgent or militaristic response. The works of the first Canon provided the basis for using the title of the response to a quarrel or a quarrel rather than a distress response. In his view, distress is the result of breaking the natural stable equilibrium when faced with an external environmental demand. Canon considers the state of equilibrium to be a relative balance of self-regulation. In his view, the human body is designed with self-regulating natural defense mechanisms. Canon was particularly interested in the role of the nervous system itself in activating humans in high-stress conditions (Ameli and Toutian, 2011).

\section{Research background}

Ismaili Nasab et al. (1393) investigated the role of difficulty in emotion regulation and emotional distress tolerance in predicting student addiction. A sample of 180 students from Allameh Tabataba'i University ( 82 males and 88 females) were selected randomly. An addiction inventory questionnaire, an emotional regulation questionnaire and a distress tolerance questionnaire were used to collect information. The results indicated that difficulty in emotion regulation and its components predicted $37.5 \%$ of addiction, and transparency was more effective among components. Also, there was no significant relationship between distress tolerance and addiction.

The results of the study, conducted by Marshall et al. (2011), which aimed to determine the mediating role of distress tolerance in impulsivity and alcohol consumption, showed that distress tolerance plays a role in this regard and these individuals play a role in relieving stress. And psychological distress is taking on alcohol.

Findings of the study Burns, Vojanick and McPherson (2010) aimed to determine the mediating role of distress tolerance in impulsivity and alcohol consumption, showed that distress tolerance plays a role in this role and these individuals play a role in relieving stress. And psychological distress to alcohol consumption

Ductres et al. (2009) conducted a study on 231 white and black American adolescents who showed low levels 
of distress, the risk of alcohol abuse among white people, delinquent behaviors among black people, and signs of disturbances Increases internalization among women.

\section{Research Methodology}

The present research in terms of the purpose of the research, typology of qualitative data, and in terms of the method was descriptive correlational. The research sample was young couples residing in Sa'adat Abad district in 2017. According to a study, about 100 young couples lived in the area. Considering sample size and referring to the Morgan table, 80 couples (40 women and 40 men) residing in Sa'adat Abad district of Tehran, ranging from 25 to 35 years old, with less 5 years marital life. they were sampled by Simple random sampling. Distress Tolerance Scale (DTS): An Extreme Distress Tolerance Self-Assessment Index that has 15 items and 4 sub-scales.

Sub-scales: tolerance (tolerance of emotional distress), absorption (absorption by negative emotions), assessment (mental distress distress) and regulation (adjustment efforts to relieve distress).

The alpha coefficients for these scales are $0.72,0.82,0.78$ and 0.70 respectively and for the whole scale is 0.82 . It is also clear that this scale has a good initial and integrative validity and validity.

Persian version of the Cognitive Critical Thinking Setup Questionnaire (CERQ-p): The Thinking Cognitive Questionnaire was developed by Garnowski, Kareije and Spinhaun in 2001 in the Netherlands and has two English and Dutch versions. This questionnaire is a self-report tool with 36 articles. The implementation of this questionnaire is very easy and can be used for people 12 years of age (both normal people and clinical population). The cognitive-emotional ordering questionnaire has a solid experimental and theoretical basis and consists of 9 sub-scales. The subscales evaluate the cognitive strategy of self-denial, acceptance, rumination, positive re-focus, re-focus planning, re-evaluation, perceptiveness, disaster, and blame for others. Scale scales range from 1 to 5. Each subscale consists of 4 items. The total score of each scorecard is obtained by aggregating the females.Hubert's 2007 Personality Register was designed to evaluate affiliated personality traits, identifying the dimensions of affective dependency in a person, and the development of affiliated personality subtypes based on DSM-IV-TR criteria. The revised version has 55 questions and two main factors (reliance on others and the need for relationships) and 8 subscales. Correct answers for score 1 and wrong answers score zero. Cronbach's alpha was 0.99 for the subscales and $0.83,0.66,0.77,0.76,0.56,0.76,53.03$ and 0.77 respectively were calculated (Mojaveroni, 2013). The analytic methods used in the descriptive statistics included the frequency of the studied variables, mean, standard error, minimum, maximum variables. The research hypotheses were also studied before the statistical analysis. The second part was related to inferential analysis of data. The basis of this study was to test the issues on the statistical analysis used in this section, which is Pearson correlation coefficient And step-bystep regression to examine hypotheses.

\section{Descriptive findings}

In the descriptive part of the descriptive data, the subjects are presented in tabular form. Then, at the inferior level of each of the hypotheses, a related statistical test is presented.

A) Sex

\section{Results}

Table 1. Frequency and percentage of research subjects by sex

\begin{tabular}{cccc}
\hline Percentage of cumulative frequency & Percentage & Frequency & Sex \\
\hline 50.0 & 50.0 & 40 & Female \\
\hline 100.0 & 50.0 & 40 & Male \\
\hline 100 & 100 & 80 & Total \\
\hline
\end{tabular}

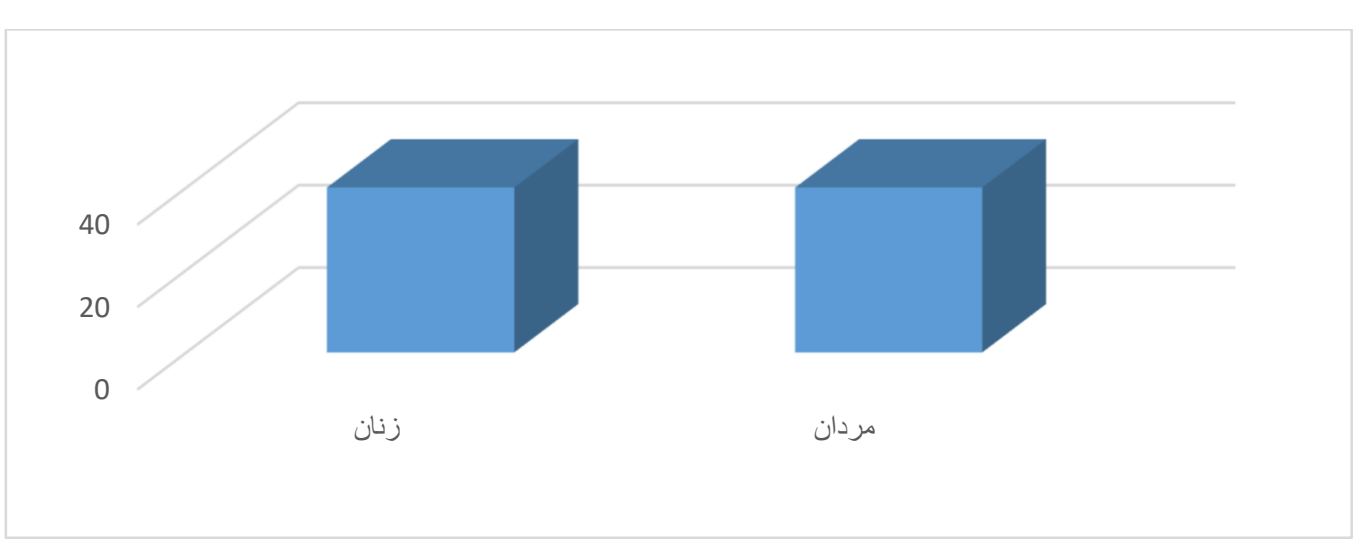

Figure 1\% Frequency of participants in research by gender 
B) age

Frequency and percentage of subjects by age were reported in the following table.

Table 2 Frequency and percentage of subjects by age

\begin{tabular}{|c|c|c|c|}
\hline Percentage of cumulative frequency & Percentage & Frequency & age categories \\
\hline $16 / 5$ & $16 / 5$ & 14 & 2927 \\
\hline 34 & $17 / 5$ & 19 & 32 تا 29 \\
\hline $50 / 5$ & $16 / 5$ & 15 & 35 تا 32 \\
\hline 68 & $17 / 5$ & 19 & 36 تا 35 \\
\hline 84 & 16 & 7 & 4036 \\
\hline 100 & 16 & 6 & 4840 \\
\hline--- & 100 & 80 & Total \\
\hline
\end{tabular}

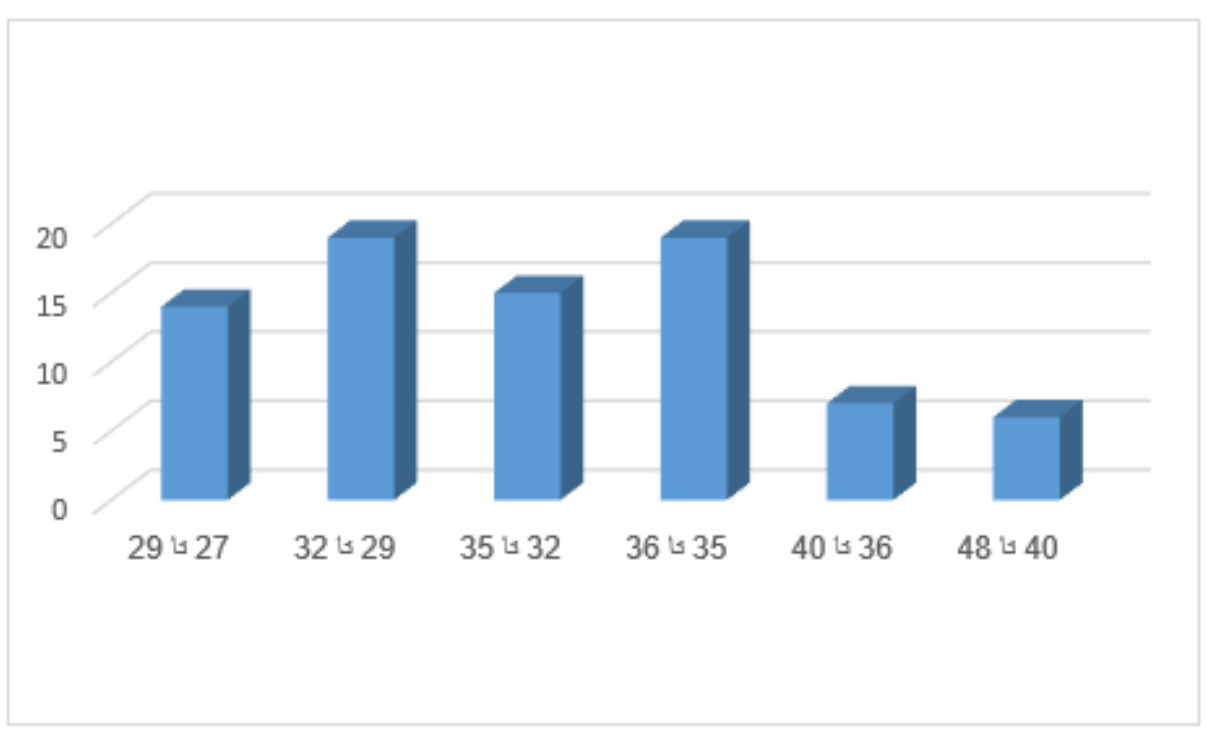

Figure 2 the frequency of participants in the research by age

According to the information obtained from the table above, the highest percentage of subjects is from age 32 to 29 and 35 to 36 , and the lowest is from age 40 to 48 .

C) Main variables of research:

In Table 3, the descriptive information of the reported variables is reported. Further, the descriptive characteristics of the main variables of the research, which include distress tolerance, emotional regulation and affiliated personality, are reported separately for the other variables in the table.

Table 3 Descriptive information (number, mean and standard deviation) of variables

\begin{tabular}{cccl}
\hline Standard Deviation & Mean & Quantity & \multicolumn{1}{c}{$\begin{array}{l}\text { Indicator } \\
\text { Variable }\end{array}$} \\
\hline $19 / 58$ & $175 / 87$ & 80 & Dependent Characteristics \\
\hline $4 / 42$ & $26 / 65$ & 80 & Distress tolerance \\
\hline $16 / 60$ & $102 / 66$ & 80 & Emotion Adjustment \\
\hline
\end{tabular}

According to the table above and the column related to the mean, the affiliated person score of 175.87 is the distress tolerance score of 26.65 and the score of the excitement score is 102.66.

\section{Inferential Findings}

First hypothesis:

There is a significant relationship between afflicted personality disorder and distress tolerance in the young couples of Sa'adat Abad in Tehran.

Table 4. Correlation matrix between dependent personality disorder and distress tolerance

\begin{tabular}{ccc}
\hline \multicolumn{3}{c}{ Distress tolerance } \\
\hline$-0 / 571^{* *}$ & The correlation coefficient & Dependent Characteristic \\
\hline $0 / 001$ & The significance level & \\
\hline
\end{tabular}

The above table is a correlation matrix between dependent personality disorder and distress tolerance. The observed correlation coefficient is -0.571 , and the significance level is 0.001 . Therefore, it can be said that the correlation coefficient score is significant at the confidence level of 0.001 . Thus, there is a meaningful negative 
relationship between dependent personality disorder and distress tolerance. Thus, the hypothesis is confirmed. Second main hypothesis: There is a significant relationship between the emotional adjustment difficulties and distress tolerance in the young couples of Sa'adat Abad in Tehran.

Table 5 Correlation matrix between difficulty setting and excitement distress

\begin{tabular}{ccccc}
\hline \multicolumn{4}{c}{ Distress tolerance } & \\
\hline$-0 / 467^{* *}$ & The correlation coefficient & $\begin{array}{l}\text { Difficulties } \\
\text { excitement }\end{array}$ & of adjusting the \\
\hline $0 / 001$ & The significance level & then
\end{tabular}

The above table is a correlation matrix between the difficulty of adjusting excitement and distress tolerance. The observed correlation coefficient is -0.467 , and the significance level is 0.001 . Thus, the correlation coefficient score is significant at the confidence level of 0.001 . Thus, there is a meaningful negative relationship between difficulty setting and distress tolerance. Thus, the hypothesis is confirmed.

The first sub hypothesis: there is a significant relationship between relying on others and enduring distress in young couples in Saadat Abad.

Table 6 Solidarity matrix between relying on others and enduring distress

\begin{tabular}{|c|c|c|}
\hline Distress tolerance & & \\
\hline${ }^{* *} 5260 /$ & The correlation coefficient & \multirow{2}{*}{ Reliance on others } \\
\hline $0 / 001$ & The significance level & \\
\hline
\end{tabular}

The above table is a correlation matrix between relying on others and distress tolerance. The observed correlation coefficient is 0.526 and the significance level is 0.001 . Thus, we can say that the correlation coefficient score is significant at the confidence level of 0.001 . Thus, there is a significant positive relationship between relying on others and tolerance of distress. Thus, the hypothesis is confirmed.

Second sub hypothesis: There is a significant relationship between the need for relationships and tolerance of distress in young couples in Sa'adat Abad.

Table 7 Correlation matrix between need for relationships and distress tolerance

\begin{tabular}{|c|c|c|}
\hline Distress tolerance & & \\
\hline ** $6230 /$ & The correlation coefficient & Need for relationships \\
\hline $0 / 001$ & The significance level & \\
\hline
\end{tabular}

The above table is a correlation matrix between the need for relationships and distress tolerance. The observed correlation coefficient is 0.623 and the significance level is 0.001 . Therefore, it can be said that the correlation coefficient score is significant at the confidence level of 0.001 . Thus, there is a significant positive relationship between the need for relationships and distress tolerance. Thus, the hypothesis is confirmed.

Third sub hypothesis: there is a significant relationship between the lack of acceptance of emotional responses and distress in young couples in Sa'adat Abad.

Table 8 Correlation matrix between acceptance of emotional responses and distress tolerance

\begin{tabular}{llll}
\hline Distress tolerance & The correlation coefficient & $\begin{array}{l}\text { Disapproving } \\
\text { responses }\end{array}$ & emotional \\
\hline $0 / 001$ & The significance level & \\
\hline
\end{tabular}

The above table is a correlation matrix between the non-acceptance of emotional responses and distress tolerance. The correlation coefficient is 0.487 and the significance level is 0.001 . Thus, the correlation coefficient score is significant at the confidence level of 0.001 . Thus, there is a meaningful positive correlation between the lack of acceptance of emotional responses and distress tolerance. Thus, the hypothesis is confirmed.

Fourth sub-hypothesis: There is a significant relationship between the difficulty in targeting behavior and endurance distress in young couples in Sa'adat Abad.

Table 9 Correlation matrix between the difficulty in performing purposeful behavior and distress tolerance

\begin{tabular}{lll}
\hline Distress tolerance & & \\
\hline${ }^{* *} 5040 /-$ & The correlation coefficient & $\begin{array}{l}\text { Difficulty in pursuing purposeful } \\
\text { behavior }\end{array}$ \\
\hline $0 / 001$ & The significance level & ran
\end{tabular}

The above table is a correlation matrix between the difficulty in performing purposeful behavior and distress tolerance. The observed correlation coefficient is 0.504 and the significance level is $0 / 001$. Thus, we can say that the correlation coefficient score is significant at the confidence level of $001 / 0$. Thus, there is a meaningful negative relationship between the difficulty in targeting behavior and distress tolerance. Thus, the hypothesis is confirmed.

Fifth sub hypothesis: There is a significant relationship between the difficulty in controlling impulse and tolerance of distress in young couples in Sa'adat Abad.

Table 10 Correlation matrix between difficulty in controlling impulse and distress tolerance

\begin{tabular}{|c|c|c|c|c|}
\hline \multicolumn{5}{|l|}{ Distress tolerance } \\
\hline ***4160/- & The correlation coefficient & \multirow{2}{*}{$\begin{array}{l}\text { Difficulty } \\
\text { momentum }\end{array}$} & \multirow[t]{2}{*}{ controlling } & the \\
\hline $0 / 001$ & The significance level & & & \\
\hline
\end{tabular}


The above table is a correlation matrix between the difficulty in controlling impulse and distress tolerance. The observed correlation coefficient is 0.416 and the significance level is 0.001 . Therefore, it can be said that the correlation coefficient score is significant at the confidence level of 0.001 . Thus, there is a significant negative relationship between difficulty in controlling impulse and distress tolerance. Thus, the hypothesis is confirmed. Sixth hypothesis: There is a significant relationship between lack of emotional awareness and distress tolerance in young couples in Sa'adat Abad.

Table 11 Correlation matrix between lack of emotional awareness and distress tolerance

Distress tolerance

\begin{tabular}{lll}
${ }^{* *} 4270 /-$ & The correlation coefficient & Lack of emotional awareness \\
\hline $0 / 001$ & The significance level & \\
\hline
\end{tabular}

The above table is a correlation matrix between the lack of emotional awareness and distress tolerance. The correlation coefficient is 0.427 and the significance level is 0.001 . Therefore, it can be said that the correlation coefficient score is significant at the confidence level of 0.001 . Thus, there is a significant negative relationship between lack of emotional awareness and distress tolerance. Thus, the hypothesis is confirmed.

Seventh hypothesis: There is a significant relationship between limited access to emotional adjustment strategies and distress tolerance in young couples in Sa'adat Abad.

Table 12 Correlation matrix between limited access to emotional adjustment and distress tolerance strategies

\begin{tabular}{llll}
\hline Distress tolerance & & \\
${ }^{* *} 5730 /-$ & The correlation coefficient & $\begin{array}{l}\text { Limited access to emotional } \\
\text { regulation strategies }\end{array}$ \\
\hline $0 / 001$ & The significance level & \\
\hline
\end{tabular}

The above-mentioned correlation matrix between access is limited to emotional adjustment and distress tolerance strategies. The observed correlation coefficient is 57/03 and the significance level of 0.001 thus, we can say that the correlation coefficient score is significant at the confidence level of 0.001 . Thus, there is a meaningful negative relationship between limited access to emotional adjustment strategies and distress tolerance. Thus, the hypothesis is confirmed.

Eighth Sub-hypothesis: There is a significant relationship between lack of clarity of emotion and distress in young couples in Sa'adat Abad.

Table 13 Correlation matrix between emotional insecurity and distress tolerance

\begin{tabular}{|c|c|c|}
\hline Distress tolerance & & \\
\hline **3500/- & The correlation coefficient & \multirow[t]{2}{*}{ Lack of emotional clarity } \\
\hline $0 / 001$ & The significance level & \\
\hline
\end{tabular}

The above table is a correlation matrix between insensitivity and distress tolerance. The observed correlation coefficient is 0.350 and the significance level is 0.001 . Therefore, the correlation coefficient score is significant at the confidence level of 0.001 . Thus, there is a meaningful negative relationship between lack of clarity of emotion and tolerance of distress. Thus, the hypothesis is confirmed.

\section{Result and Conclusion}

The first main hypothesis: There is a significant relationship between dependent personality disorder and distress tolerance in the young couples of Sa'adat Abad in Tehran. The results of this study showed that there was a significant negative correlation between dependent personality disorder and distress tolerance in the young couples of Sa'adat Abad, according to the significance level and the correlation coefficient. This means that with the increase in dependent personality disorders in couples, tolerance of distress in them should be reduced. This finding is consistent with the results of Oberke et al. (2007). In the context of explanation, it can be said that people who are afflicted with affliction personality are extremely dependent on others and constantly need others' confidence, lack of tolerance of loneliness and distress, and the high sensitivity to being rejected in them. It can be seen that they agree with others too, in other words, they do not dare to disagree with others.

The second main hypothesis: There is a significant relationship between the difficulties of emotion regulation and distress tolerance in the young couples of Sa'adat Abad in Tehran. The results of this study showed that there is a significant negative correlation between emotional adjustment difficulties and distress tolerance in the young couples of Sa'adat Abad, according to the significance level and the correlation coefficient. This means that with the increasing difficulty of adjusting the excitement in couples, they should increase their distress. This finding is in line with the results of Putra et al. (2011) and Marshall et al. (2011), Burns, Vojanick and McPherson (2010), Dacters et al. (2009), and Simon and Gaher (2012). In order to explain, it can be said that the possibility of tolerance of low distress alone can be a factor in the occurrence of physical problems, especially severe headache. People with tension headache respond to avoidance behaviors to control the stressors and react to it by escaping stressful situations. On the contrary, those who have high levels of intolerance, while embracing their excitement, seek immediate relief from or avoidance of negative emotions.

The first sub-hypothesis: There is a significant relationship between relying on others and enduring distress 
in young couples in Sa'adat Abad.

The results of this study showed that there is a significant positive correlation between the reliance on others and distress tolerance in the young couples of Saadat Abad, according to the significance level and the correlation coefficient. This means that increasing reliance on others in couples should increase their distress. This finding is in line with the results of Putra et al. (2011) and Marshall et al. (2011), Burns, Vojanick and McPherson (2010), Dacters et al. (2009), and Simon and Gaher (2012). In order to explain, it can be said that reliance on others is one of the symptoms of affiliated personality disorder. Individuals with a reliance on others have the ability to make decisions unless they consult with others and are fully confident in each case that their decision is correct.

The second sub-hypothesis: There is a significant relationship between the need for relationships and tolerance of distress in young couples in Sa'adat Abad. The results of this study showed that there is a significant positive correlation between the need for relationships and tolerance of distress in the young couples of Saadat Abad, according to the significance level and the correlation coefficient. This means that increasing the need for relationships in couples should increase their distress. This finding is in line with the results of Putra et al. (2011) and Marshall et al. (2011), Burns, Vojanick and McPherson (2010), Dacters et al. (2009), and Simon and Gaher (2012). In order to explain, it can be said that communicating between couples is the main and most important issue in human life, especially in marital life, but in the case of people who have a personality, this type of relationship goes out of the ordinary and becomes a severe need for conversion. Because the person does not feel distressed or stressed.

The third sub-hypothesis: there is a significant relationship between the lack of acceptance of emotional responses and distress in young couples in Sa'adat Abad. The results of this study showed that there is a significant positive correlation between the lack of acceptance of emotional responses and distress tolerance in the young couples of Saadat Abad, according to the significance level and the correlation coefficient. This means that with the increasing rejection of emotional responses in couples, tolerance of distress in them should increase. This finding is in line with the results of Putra et al. (2011) and Marshall et al. (2011), Burns, Vojanick and McPherson (2010), Dacters et al. (2009), and Simon and Gaher (2012). In order to explain, it can be said that as many individuals in the family environment develop their emotions and express their feelings and be able to freely discuss and discuss in a wide range of topics, they can resilience and tolerance There is more distress in the future, and vice versa, when one is not able to accept emotions from others, he quickly becomes distressed and worried about.

The fourth sub-hypothesis: There is a significant relationship between the difficulty in targeting behavior and endurance distress in young couples in Sa'adat Abad. The results of this study showed that there is a significant negative relationship between the difficulty in performing purposeful behavior and distress tolerance in the young couples of Saadat Abad, according to the significant level score and the correlation coefficient. It means that with increasing difficulty in carrying out purposeful behavior in couples, tolerance of distress in them should be reduced. This finding is in line with the results of Putra et al. (2011) and Marshall et al. (2011), Burns, Vojanick and McPherson (2010), Dacters et al. (2009), and Simon and Gaher (2012). In order to explain, one can say that those who cannot fulfill their planned behaviors properly and whose expectations have not been met are worried and stressed and those who have a personality who are dependent on fear of abusing and rejecting others, Are often reactive and spontaneously spontaneous.

The fifth sub-hypothesis: There is a significant relationship between the difficulty in controlling impulse and tolerance of distress in young couples in Sa'adat Abad. The results of this study showed that there is a significant negative relationship between the difficulty in performing purposeful behavior and distress tolerance in the young couples of Saadat Abad, according to the significant level score and the correlation coefficient. It means that with increasing difficulty in carrying out purposeful behavior in couples, tolerance of distress in them should be reduced. This finding is in line with the results of Putra et al. (2011) and Marshall et al. (2011), Burns, Vojanick and McPherson (2010), Dacters et al. (2009), and Simon and Gaher (2012). In order to explain, one can say that people who are incapacitated and able to withstand a momentum or motive that is dangerous to others or themselves are always in an aggressive state and do not have the necessary focus and can cope with problems and problems.

The sixth sub-hypothesis: There is a significant relationship between lack of emotional awareness and distress tolerance in young couples in Sa'adat Abad. The results of this study showed that there is a significant negative relationship between the lack of emotional awareness and distress tolerance in the young couples of Saadat Abad, according to the significance level and the correlation coefficient. This means that with the increasing lack of emotional awareness in couples, tolerance of distress in them should be reduced. This finding is in line with the results of Putra et al. (2011) and Marshall et al. (2011), Burns, Vojanick and McPherson (2010), Dacters et al. (2009), and Simon and Gaher (2012). In terms of explanation, it can be said that people who are lacking in emotional awareness are unaware of their inner feelings and are constantly unaware of their state of affairs and their mental and psychological conditions, and do not know what is happening at this moment. Turmoil. When needed, people need to pay attention to their inner heart and heart, but these people are confused and do not pay attention to their true emotions, and this causes distress in the individual and distributes this distress to others 
around them.

The seventh sub-hypothesis: There is a significant relationship between limited access to emotional adjustment strategies and distress tolerance in young couples in Sa'adat Abad. The results of this study showed that there is a significant negative relationship between limited access to emotional adjustment strategies and distress tolerance in the young couples of Sa'adat Abad, according to the significance level and the correlation coefficient. This means that by increasing the limited access to emotional regulation strategies in couples, tolerance to distress should be reduced. This finding is in line with the results of Putra et al. (2011) and Marshall et al. (2011), Burns, Vojanick and McPherson (2010), Dacters et al. (2009), and Simon and Gaher (2012). In order to explain, it can be said that when a person is exposed to an unpleasant stimulus and is left unprotected by his or her side, it is distressed, which causes weak management of emotions and regulation, and against those who are regulated Have a high positive emotion, they are more likely to predict the wishes of others.

The eighth sub-hypothesis: There is a significant relationship between lack of clarity of emotion and tolerance of distress in young couples in Sa'adat Abad. The results of this study showed that there is a significant negative correlation between emotional insecurity and distress tolerance in the young couples of Saadat Abad according to the significance level and the correlation coefficient. This means that increasing the lack of clarity of emotions in couples should reduce their distress. This finding is in line with the results of Putra et al. (2011) and Marshall et al. (2011), Burns, Vojanick and McPherson (2010), Dacters et al. (2009), and Simon and Gaher (2012). In order to explain, it can be said that many theorists believe that emotional maladaptation plays a central and important role in the causation and development of personality disorder, which increases emotional insecurity and anxiety and anxiety. The emotional regulation is called the regularization and adjustment of emotional processes, so the non-regulation means irregularities of emotion.

\section{References}

Aldao, A., Nolen-Hoeksema, S., \&Schweizer, S. (2010). Emotion- regulation strategies across psychopathology: A meta analytic review. Clinical Psychology Review, 30, 217-237.

Marshall-Berenz, E. C., Vujanovic, A. A., MacPherson, L. (2011). Impulsivity and alcohol use coping motives in a trauma-exposed sample: The mediating role of distress tolerance. Personality and Individual Differences, $50,588-592$.

Pottera, C. M., Vujanovic, A. A., Marshall-Berenz, E. C., Bernsteind, A., Bernsteind, M. O. (2011). Posttraumatic stress and marijuana use coping motives: The mediating role of distress tolerance. Journal of Anxiety Disorders, 25, 437-443.

Simons.J.S and Gaher, R. M (2005). The distress tolerance scale: Development \& Validation pf a self report measure. Motivation \& emotion. 20(9),83-102.

Atkinson R. (1999). The Hylgard Psychology. C 1, translation by Dr. Barahini et al. Grow Publishing

Isma'ili Nasab, Maryam, Kermanshah, Alireza, Azrami, Haleh, Shemarrekhi, Amir. (1393). The role of predictor of difficulty in regulation of emotion and distress tolerance in students' addiction, Addiction Research, Volume 8, Issue 29, Pages 49-63.

Rio, John Marshall (2006) Motivation and Excitement, Yahya Seyyed Mohammadi translation, Tehran. Publish edited.

Seyyed Mahdavi Aghdam, Mirrullah, Mahmoodalilou, Majid, Rezaei, Omid. (1392). Relationship between different experiences of child abuse and personality disorders in cluster B, Medical Journal of Urmia University of Medical Sciences, Volume 24, Issue 3, Pages 210-278.

Azizi, Alireza, Mirzaei, Azadeh, Shams, Jamal (2010). Investigating the Tolerance of Distress and Emotional Settlement with the Level of Students' Dependence on Cigarettes, Hakim Research Journal, Thirteenth, No. 1, pp. 11-18.

Mohammadi, Narges, Ali Pour, Ahmad, Doqaghzadeh, Hamed, Farzad, Valiyah (1394). Comparison of emotional regulation difficulties in patients with irritable bowel syndrome with regard to handicap, behavioral science research, Volume 12, Issue 4. 